\title{
Development of multiple plug-in electric vehicle mobile charging station using bidirectional converter
}

\author{
A. Dominic Savio ${ }^{1}$, Vimala Juliet A. ${ }^{2}$ \\ ${ }^{1}$ Department of Electrical and Electronics Engineering, SRM Institute of science and technology, India \\ ${ }^{2}$ Department of Electronics and Instrumentation Engineering, SRM Institute of science and technology, India
}

\begin{tabular}{l} 
Article Info \\
\hline Article history: \\
Received Mar 10, 2019 \\
Revised Jul 8, 2019 \\
Accepted Dec 6, 2019 \\
\hline
\end{tabular}

Keywords:

DC Nanogrid

Electric Vehicles

Mobile charging station

Vehicle to grid

\begin{abstract}
Electric vehicle (EV) charging station powered by the scattered energy sources with DC Nanogrid (NG) provides an option for uninterrupted charging. The NG powered by the renewable energy sources (RES) of photovoltaic (PV) and wind energy. When the excess power produced by the renewable energy stored in the local energy storage unit (ESU) utilized during shortage power from the renewable sources. During the overloading of NG and demand of energy in ESU; the mobile charging station (MCS) provides an uninterrupted charging. The MCS provides an option for battery swapping and vehicle to grid feasibility. The MCS required to monitor the state of charge (SOC) and state of health ( $\mathrm{SOH}$ ) of the battery. Monitoring of SOC and SOH related to the various battery parameters like voltage, current and temperature. A laboratory prototype is developed and tested the practical possibility of EV to NG and Internet of things (IoT) based monitoring of battery parameters.
\end{abstract}

This is an open access article under the CC BY-SA license.

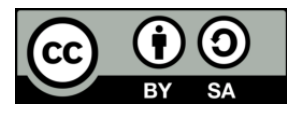

\section{Corresponding Author:}

A. Dominic Savio,

Department of Electrical and Electronics Engineering,

SRM Institute of science and technology,

Chennai 603203, Tamil Nadu, India.

Email: agdominicsavio@gmail.com

\section{INTRODUCTION}

Depletion of fossil fuel, increasing fuel prices and long term benefit of pollution free environment all addressed by the rode transportation electrification [1]. In the transportation sector electric vehicles (EVs) provide an option for pollution-free road transportation [2]. In order to minimize the dependency of conventional energy source based EV charging and reducing stress on the existing utility grid renewable energy sources integrated with the charging station architecture. When there is an excess power produced by renewable energy sources: stored in the energy storage unit (ESU) [3, 4]. During the shortage of energy for EV charging or peak demand in the utility grid, ESU or fully charged EV supplies power to EV charging [5].

The major disadvantage in the conversion road transportation in to electrical is charging time and battery life [6]. The MCS integrated with the NG or home, reducing the overloading of the utility grid also energy management system provides better power management in the charging station [7, 8]. Also, the service centre able to provide support for MCS for uninterrupted driving [9]. The power electronic converter, along with a suitable pulse width modulation control provides an efficient charging system performance [10, 11]. The proposed charging station topologies consist of a DC-DC buck converter for EV charge, DC-DC boost converter for grid integration [12-14]. The MCS with battery storage system with bidirectional DC-DC buck/boost parallel converters offers a bidirectional charging option. 
The MCS SOC and SOH are measured from the coulomb counting method for the estimation of SOC stands mid-way between kalman filter and OCV-SOC method [15]. This method involves the counting of ampere-hours that takes part in the whole process of charging and discharging in a battery. It takes into consideration the determination of initial SOC which is of great importance since it is responsible for the later calculated SOC at any instant [16]. For estimating initial SOC we may make use of OCV-SOC curve from a proposed piecewise linear relationship of SOC-OCV done by some of the experts [17]. Better way to get the initial SOC is by operating the battery in three modes charging, discharging and open-circuited mode and by using each mode the charging voltage, current and estimation curves are drawn and then out of those curves we try to get equations relating SOC and charging discharging current or sometimes voltages [18].The SOC is then estimated by considering other important factors also like SOH (State of health), DOD (Depth Of Discharge), and a much more important parameter Charging and Discharging efficiencies as is used in an improved or enhanced coulomb counting method [15]. The use of charging and discharging efficiencies is beneficial for increasing the overall accuracy of the system and thus reducing the chances of error. This method is easy to implement and gives accurate result as compared to OCV-SOC technique but less accurate as compared to kalman filter SOC estimation technique. This method is very accurate if the initial SOC, current, and efficiency is precise [19]. The only issue with this method is that it needs an accurate initial SOC which is difficult to get and therefore sometimes the accuracy is less.

However, battery swapping more reliable for long-distance travel, different problem related to the battery swapping is standardizing the battery type continuous health monitoring $[20 ; 21]$. To improve the battery swapping cloud-based battery parameters storage is used. Therefore, using IoT within the electric vehicle charging station will make it easier [22]. An autonomous system will be designed for fault detection in electric vehicle and transmit fault data to the server [23]. The cloud and based on information, it sends respective notifications to the driver as well as the MCS [24]. It will help the service team to solve the problem at the site itself, thus increasing efficiency as well as reducing time and cost involved.

The paper is structured as follows the architecture of multiport electric vehicle charging station explained in secession 2. Various modes of charging stations with battery swapping explained in section 3 . The simulation is performed using MATLAB/Simulink, and the results were presented in section 3 . Experimental validation of simulation results discussed in section 4 . Conclusion, the advantages of the proposed system is given in Section 5.

\section{RESEARCH METHOD}

The multiport electric vehicle smart charging station architecture with MCS is shown in taure 1 . The charging station uses common DC NG to power charging point and the local load. The NG powered by the different PV sources, wind generator and onboard energy storage unit All the sources are connected through the power electronics converter; PV connected to the NG through maximum power point tracking (MPPT) boost converter [25, 26]. The MPPT controller provides maximum output from the PV. The different charging terminals are connected through the power electronics converter [27]. Some charging points are used a bidirectional converter to operate vehicle to other modes. The local load connected to the charging station considered as a DC house for avoiding losses. The MCS is associated with the changing station when there is demand. The isolation switches are connected between the load terminal and NG to decide the smart charging modes.

When a fault occurs in an electric vehicle and battery, it gets immobilized and this causes a lot of problem for the owner. Hence the vehicle or battery swapping service provider continuously monitors the battery parameters. Therefore, using IoT within the electric vehicle system will make it easier. An autonomous system will be designed for fault detection in electric vehicle and transmit fault data and battery parameters to the MCS. It will help the service team to solve the problem at the site itself thus increasing efficiency as well as reducing time and cost involved.

Developing a smart charging station with the facility of battery swapping and vehicle to grid provides a following.

- Vehicle to vehicle charging power transferred from number of EV to required EV through the NG DC bus via bidirectional converter reducing the stress on NG.

- $\quad \mathrm{MEV}$ to grid charging mobile electric vehicle provides a power to the NG to charge the available EVs at the charging station.

- MCS can afford a battery swapping based on the parameters monitored through the IoT; swapping charges are calculated.

Int J Pow Elec \& Dri Syst, Vol. 11, No. 2, June 2020 : $785-791$ 


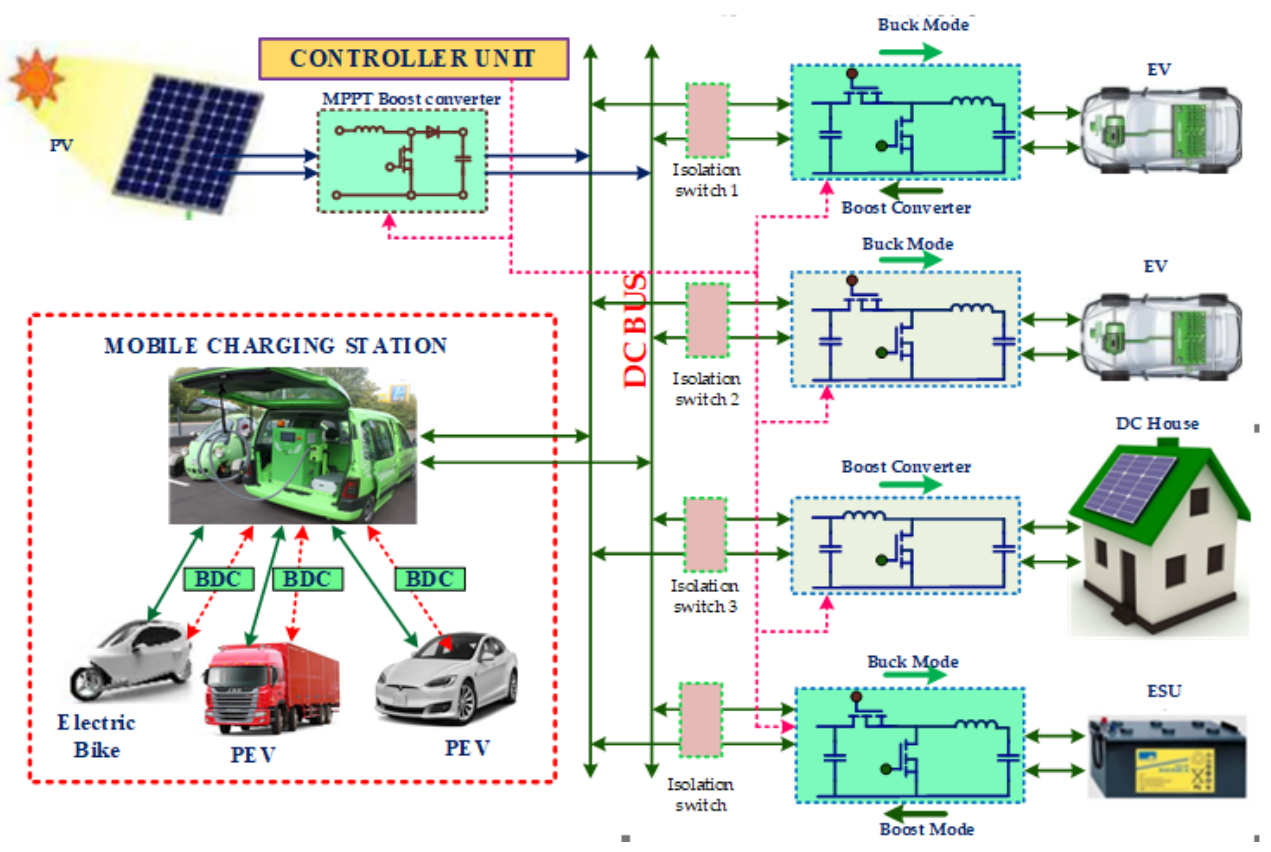

Figure 1. Charging station architecture

The battery swapping station monitors current, voltage and temperature etc. For the calculation of performance of the battery and useful information of SOC and SOH of the battery transferred through the IOT. Based on this parameters controlling action, pricing range of battery swapping and battery life cycle are measured. The charging station and the MCS are operated in a different mode, basically three different modes are described. All this mode is based on the voltage level of DC NG and SOC of MCS charging station. When the DC bus voltage measure is less than the reference level charging station totally depends on the MCS and EVs available at the charging station. SOC of the MCS charging station greater than the defined level, it powers the NG to charge the EV else it will offer battery swapping. Modes of operation of the charging system as shown in Figure 2.

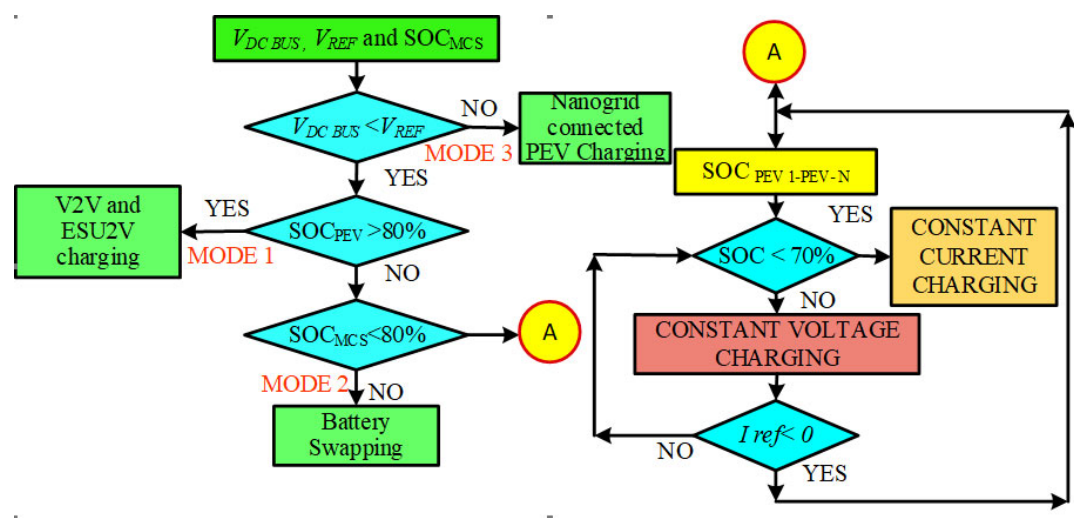

Figure 2. Modes of operation of the charging system

Vehicle to grid technology gives the power to the grid from the vehicle available charging point when the grid under peak demand for electricity. During this time, the relevant information can be transferred from the vehicle to the control centre. In this vehicle to grid technology, the number of vehicles is joined together to provide bidirectional power and communication signal. 


\section{SIMULATION RESULTS}

During simulation analysis three EVs considered for charging and discharging and the SOC values are selected as different level. The initial value of SOC while connecting to the charging station is considered as $60 \%, 70 \%$ and $80 \%$ respectively During MCS discharging mode the DC bus voltage maintained as $320 \mathrm{~V}$ through the mobile EV charging unit is shown in Figure 3. In this mode DC bus voltage is maintained and the bidirectional converter connected at the charging terminal operated in boost mode to discharge the MCS and the corresponding voltage of MCS is $48 \mathrm{~V}$ shown in Figure 4.

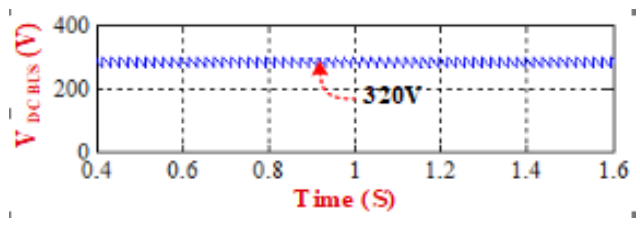

Figure 3. DC bus voltages charging mode Figure

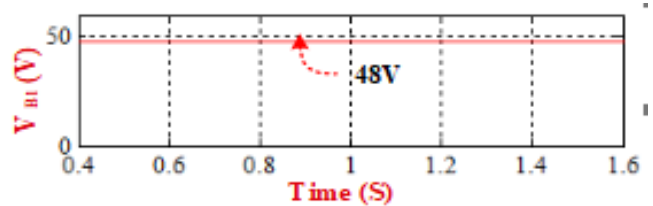

Figure 4. Charging voltage of BDC under buck mode

During vehicle to grid and ESU to Vehicle mode; ESU are vehicles available at the charging points gives the supply to the grid through the MCS. When only one vehicle is connected to discharging DC bus voltage maintained as $220 \mathrm{~V}$ and all the vehicles and ESU available at the charging point is discharging and the corresponding DC bus voltage maintained as $300 \mathrm{~V}$ is shown in Figure 5. The current and current injected to the grid is shown in Figure 6.

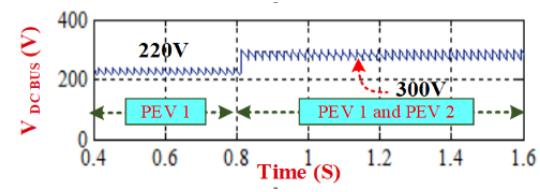

Figure 5. Vehicles discharging.

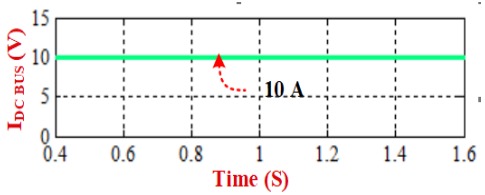

Figure 6. Feedback grid current

The vehicle to grid technology provides the technical feasibility and its best for the EV owner in economic incentive. Based on the voltage and SOC value battery, swapping mode can be selected. we can notice that the trend of SOC with respect to time is almost similar with a slight error with the estimation method. This error is a result of the enhanced coulomb counting method which gives a small error difference but is still very accurate as compared to OCV-SOC method of estimation and thus it can be considered. In order to reduce this error, it will have to monitor properly factors that affect the state of health of our battery and we can achieve more accurate results.

\section{EXPERIMENTAL VALIDATION}

The charging station with a three charger with three charging port charging station takes time to charge EV. In multiple charger, multiport charging provides a good option for mobile charging, due to its different range of charging. For the multiple electric vehicles charging different power rating are chosen for the power converter: power converters, dc grid and a digital controller. Three PEVs (PEV1, PEV2 and PEV3) are connected to the NG through DC-DC buck converter assuming that all the PEVs at different power levels of SOC, the maximum DC bus voltage is $320 \mathrm{~V}$. The voltage rating of the SBs are: SB $1=12.1$ $\mathrm{V}$, SB $2=12.7 \mathrm{~V}$ and SB $3=12.3 \mathrm{~V}$. The experimental results are presented for different operating conditions of the charging system, such as grid to the vehicle $(\mathrm{G} 2 \mathrm{~V})$ and Vehicle to grid (V2G). The laboratory prototype of $48 \mathrm{~V}, 50 \mathrm{Ah}$ battery bank used as ESU and charging system consisting of a digital controller. The photograph of the experimental setup is shown in Figure 7.

Int J Pow Elec \& Dri Syst, Vol. 11, No. 2, June 2020 : 785 - 791 


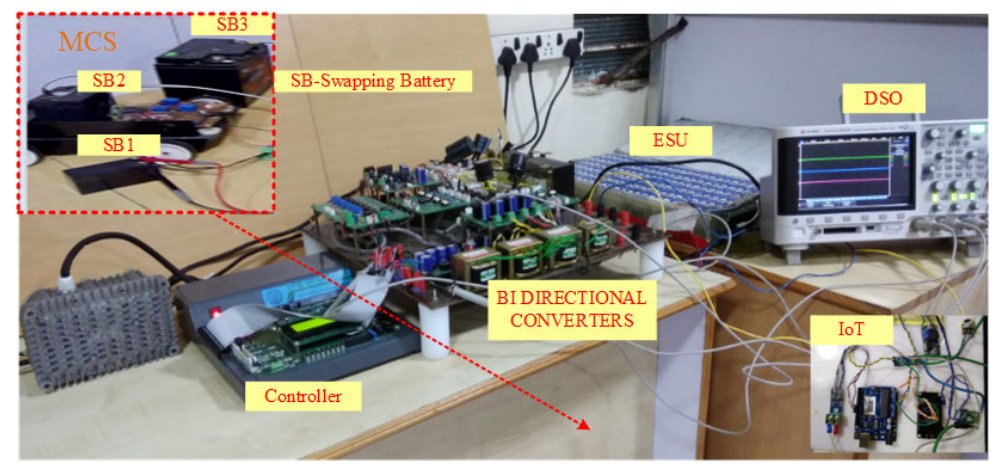

Figure 7. Experimental setup of charging station

A digital controller generates the control signals for the power electronics converters. The experimental result of all operation of the system is shown. The DC bus voltage maintained as the $48 \mathrm{~V}$ and the SBs are charged with the 12 with the switching current of the converter is $1.5 \mathrm{~A}$. From the results, it is observed that the EV charging system is functioning satisfactorily in MCS to PEV charging mode. During vehicle to vehicle and vehicle to mobile electric vehicle ESU charging mode the DC bus voltage is maintained as $12 \mathrm{~V}$. Swapping battery voltage and ESU voltages are measured is shown in Figure 8 . In this mode, All the PEVs are fully charged and the charging station total power given to the ESU. Figure 9 shows the MCS to grid powering mode, wherein this mode $48 \mathrm{~V}$ is given to the ESU.

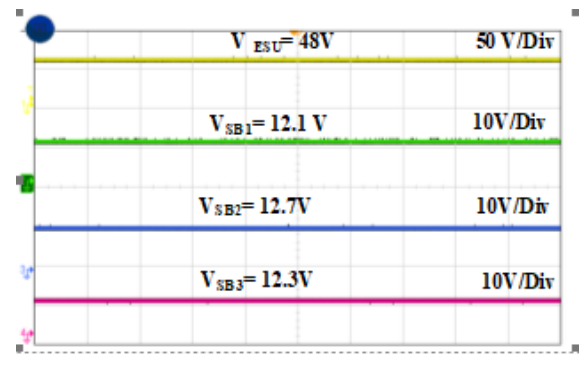

Figure 8. Experimental result of SB and ESU voltage

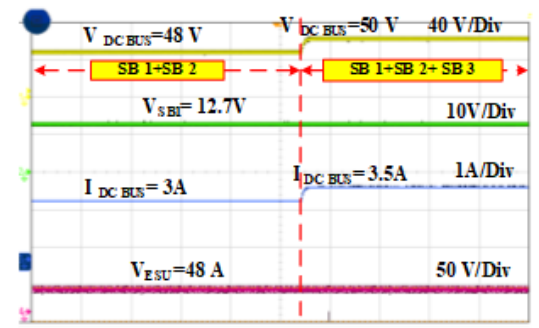

Figure 9. Experimental result ofMCS to G charging

The different modes of PEV charging station are developed and the function of the system is tested for the modes like G2V, MCS $2 \mathrm{G}, \mathrm{V} 2 \mathrm{~V}$ and battery swapping are verified. In all the modes DC bus voltage are maintained in all the modes. The simulation values are verified with the experimental setup.

For the IoT based measurement data collected by the DAQ system to the cloud. Thingspeak cloud space has been used for storing as well as computing on the data sent. At thingspeak channel real-time processing of the data can be done all the necessary real-time data has been sent. Which The data sent is being processed to estimate $\mathrm{SoC}$ and $\mathrm{SoH}$. The field one two and three are the sent data, and the field four and five are the processed outputs of SoC and SoH. The field seven is the initial slope which the system is feed for the first run and as the processing goes on the system changes it based on iterations. The initial slope is updated with the value of the slope of Ah and Voltage linear fit locus of the first iteration when a fresh battery is added to the system is shown in Figure 10a and 10b. The rises and drops of temperature are also being monitored and if the temperature reaches beyond threshold a notification system has been designed which can be used to either send caution to the battery swapping is shown in Figure 10c. 


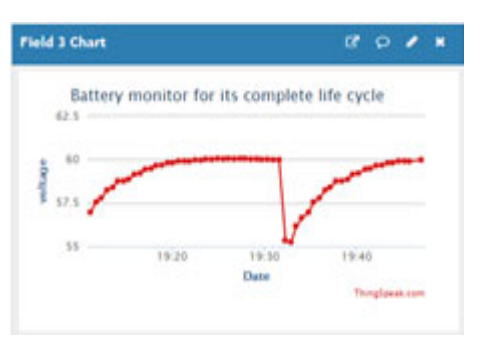

(a)

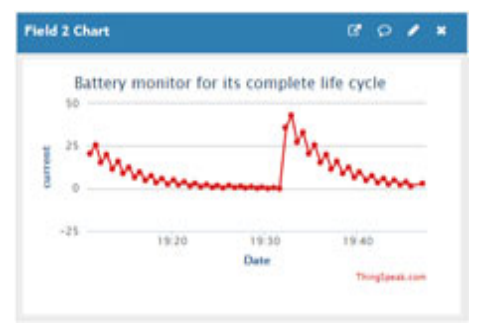

(b)

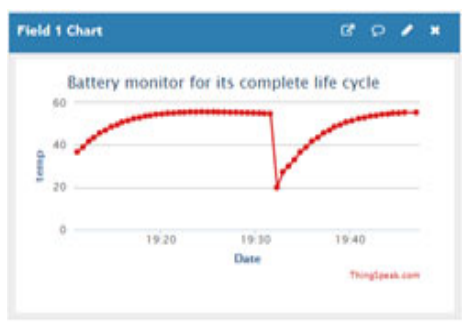

(c)

Figure 10. (a) Voltage of the battery, (b) Current of battery (c), The temperature of the battery

The channel gives us the information regarding battery voltage, current drawn, $\mathrm{SoC}, \mathrm{SoH}$, as well as the battery pack temperature required for the safe operation of the battery and swapping details. The thingspeak cloud dashboard where we had sent our real-time data acquired from the data acquisition system. The figures show the changes in the battery voltage, temperature and current with time. The battery data is stored on the channel, which is used for monitoring as well as analyzing. Whenever any abnormality occurs the notification to the mobile electric vehicle charging station.

\section{CONCLUSION}

In this paper, Electric vehicle (EV) charging system integrated with DC NG and nonconventional energy sources has been designed and developed. The idea is to transmit faults of the battery from the vehicle to the server as they arise and also predict the upcoming faults and battery parameters. The battery parameters are measured as a state of charge $(\mathrm{SoC})$, state of health $(\mathrm{SoH})$ and battery temperature used for battery swapping. Also, it is required for swapping like SoC and $\mathrm{SoH}$ estimation methods and other fault data. Also, the work includes the details about server platform and Internet of Things (IoT). Mobile electric vehicle charging station contains a fully charged batteries and the battery swapping parameters described in detail. The implementation of $\mathrm{V} 2 \mathrm{G}$ and MCS $2 \mathrm{NG}$ support is tested using a laboratory prototype.

\section{REFERENCES}

[1]. Lave, Lester B., and Heather L. MacLean, "An environmental-economic evaluation of hybrid electric vehicles: Toyota's Prius vs. its conventional internal combustion engine Corolla."Transportation Research Part D: Transport and Environment, Vol. 2, pp.155-162, 2002.

[2]. Ortmeyer, Thomas H. and Pragasen Pillay, "Trends in transportation sector technology energy use and greenhouse gas emissions." Proceedings of the IEEE 89, Vol. 12, pp. 1837-1847, 2001.

[3]. Thackeray, et al., "Electrical energy storage for transportation — approaching the limits of, and going beyond, lithium-ion batteries." Energy \& Environmental Science Vol. 5, no. 7, pp. 7854-7863, 2012.

[4]. Köhler, U, et al., "High performance nickel-metal hydride and lithium-ion batteries." Journal of power sources Vol. 105, no. 2, pp. 139-144, 2002.

[5]. Wael A. et al., "Electric vehicle technology impacts on energy" International Journal of Power Electronics and Drive System (IJPEDS) Vol. 10, No. 1, pp. 1-9, 2019.

[6]. Strunz, Kai, et al., "DC NG for wind and solar power integration." IEEE Journal of emerging and selected topics in Power Electronics, Vol. 2, no. 1, pp. 115-126, 2014.

[7]. Tie, S.F. and Tan, C.W, "A review of energy sources and energy management system in electric vehicles". Renewable and sustainable energy reviews, no. 20, pp. 82-102, 2013.

[8]. Savio, Dominic A. et al., "Photovoltaic Integrated Hybrid NG Structured Electric Vehicle Charging Station and Its Energy Management Approach." Energies, Vol. 1, no 12, pp. 168, 2019.

[9]. Mak, Ho-Yin, Ying Rong, and Zuo-Jun Max Shen. "Infrastructure planning for electric vehicles with battery swapping." Management Science Vol. 59, no. 7: 1557-1575, 2013.

[10]. Konstantinou, Georgios S., and Vassilios G. Agelidis. "Performance evaluation of half-bridge cascaded multilevel converters operated with multicarrier sinusoidal PWM techniques." 4th IEEE Conference on Industrial Electronics and Applications, pp. 3399-3404. IEEE, 2009.

[11]. Baroudi, Jamal A., Venkata Dinavahi, and Andrew M. Knight. "A review of power converter topologies for wind generators." Renewable energy Vol. 32, no. 14: 2369-2385, 2007.

[12]. Camara, Mamadou Baïlo, Hamid Gualous, Frederic Gustin, Alain Berthon, and Brayima Dakyo. "DC/DC converter design for supercapacitor and battery power management in hybrid vehicle applications-Polynomial control strategy." IEEE Transactions on Industrial Electronics Vol. 57, no. 2: 587-597, 2009.

Int J Pow Elec \& Dri Syst, Vol. 11, No. 2, June 2020 : 785 - 791 
[13]. Gu, Bin, Jason Dominic, Jih-Sheng Lai, Zheng Zhao, and Chuang Liu. "High boost ratio hybrid transformer DCDC converter for photovoltaic module applications." IEEE Transactions on Power Electronics Vol. 28, no. 4: 2048-2058, 2012.

[14]. Rehman, Zubair, Ibrahim Al-Bahadly, and Subhas Mukhopadhyay. "Multiinput DC-DC converters in renewable energy applications-An overview." Renewable and Sustainable Energy Reviews Vol. 41: 521-539, 2015.

[15]. Ng, Kong Soon, Chin-Sien Moo, Yi-Ping Chen, and Yao-Ching Hsieh. "Enhanced coulomb counting method for estimating state-of-charge and state-of-health of lithium-ion batteries." Applied energy Vol. 86, no. 9: 1506-1511, 2009.

[16]. Codeca, Fabio, Sergio M. Savaresi, and Giorgio Rizzoni. "On battery state of charge estimation: A new mixed algorithm." IEEE International Conference on Control Applications, pp. 102-107. IEEE, 2008.

[17]. Waag, Wladislaw, Christian Fleischer, and Dirk Uwe Sauer. "Critical review of the methods for monitoring of lithium-ion batteries in electric and hybrid vehicles." Journal of Power Sources Vol. 258: 321-339, 2014.

[18]. Plett, Gregory L. "Extended Kalman filtering for battery management systems of LiPB-based HEV battery packs: Part 3. State and parameter estimation." Journal of Power sources Vol. 134, no. 2: 277-292, 2004.

[19]. Xiong, Rui, Hongwen He, Fengchun Sun, and Kai Zhao. "Evaluation on state of charge estimation of batteries with adaptive extended Kalman filter by experiment approach." IEEE Transactions on Vehicular Technology Vol. 62, no. 1: 108-117, 2012.

[20]. Mak, Ho-Yin, Ying Rong, and Zuo-Jun Max Shen. "Infrastructure planning for electric vehicles with battery swapping." Management Science Vol. 59, no. 7: 1557-1575, 2013.

[21]. Tan, Xian Qiu, Sheng Chun Yang, Yan Ping Fang, and Dong Xue. "Discussion on Operation Modes to the Electric Vehicle Charging Station." In Advanced Materials Research, Vol. 875, pp. 1827-1830, 2014.

[22]. Akin, Bilal, Salih Baris Ozturk, Hamid A. Toliyat, and Mark Rayner. "DSP-based sensorless electric motor faultdiagnosis tools for electric and hybrid electric vehicle powertrain applications." IEEE Transactions on Vehicular Technology Vol. 58, no. 6: 2679-2688, 2009

[23]. Wu, Yunna, Meng Yang, Haobo Zhang, Kaifeng Chen, and Yang Wang. "Optimal site selection of electric vehicle charging stations based on a cloud model and the PROMETHEE method." Energies Vol. 9, no. 3: 157, 2016.

[24]. Wang, Feng, Xinke Wu, Fred C. Lee, Zijian Wang, Pengju Kong, and Fang Zhuo. "Analysis of unified output MPPT control in subpanel PV converter system." IEEE Transactions on power Electronics Vol. 29, no. 3: 12751284, 2013.

[25]. Sun, Kai, Li Zhang, Yan Xing, and Josep M. Guerrero. "A distributed control strategy based on DC bus signaling for modular photovoltaic generation systems with battery energy storage." IEEE Transactions on Power Electronics Vol. 26, no. 10: 3032-3045, 2011.

[26]. Zhou, Haihua, Tanmoy Bhattacharya, Duong Tran, Tuck Sing Terence Siew, and Ashwin M. Khambadkone. "Composite energy storage system involving battery and ultracapacitor with dynamic energy management in microgrid applications." IEEE transactions on power electronics Vol. 26, no. 3: 923-930, 2010.

[27]. Chiu, Yi, and Victor FG Tseng. "A capacitive vibration-to-electricity energy converter with integrated mechanical switches." Journal of Micromechanics and Microengineering, Vol. 18, no. 10: 104004, 2008.

\section{BIOGRAPHIES OF AUTHORS}

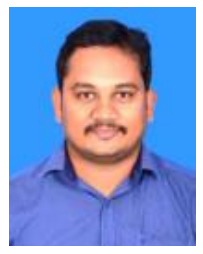

A. Dominic savio received the B.E. degree in Electrical and Electronics engineering from Anna University, in 2007, and the M.Tech degrees in control and instrumentation engineering from karunya University, Coimbatore, in 2010. He is currently pursuing the Ph.D. degree with SRM University, India. Since 2010, he has been working as an Assistant Professor with SRM Institute of Science and Technology. His research interests include electric vehicle charging infrastructure, electric vehicle charging converter, power management in electric vehicle charging infrastructure.

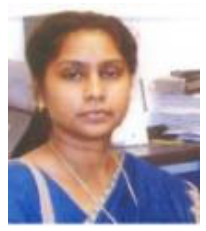

A. Vimala Juliet received the B.E. degree in electronics and instrumentation engineering from Bharathiar University, Coimbatore, in 1992, and the M.E. and Ph.D. degrees in electronics and instrumentation engineering from Anna University, Chennai, in 1994 and 2005, respectively. She started her career as a Research Engineer Trainee at FCRI, India. She was a Lecturer with a private engineering college for one year. Since 1995, she has been with the Department of Electronics and Instrumentation Engineering, SRM Institute of Science and Technology, Kattankulathur, where she is currently a Professor and the Head of the department 